\title{
Algunas limitaciones en la comprensión de la economía colaborativa en sentido emancipador
}

\section{Some limitations in understanding the collaborative economy in an}

\section{emancipatory sense}

\section{*Bru Laín}

Departamento de Sociología. Universidad de Barcelona. España/Spain

bru.lain@ub.edu

\section{RESUMEN}

¿Cómo comprender la economía colaborativa? ¿Qué definición empleamos que sea metodológicamente operativa a la vez que sustancialmente informativa? Identificando las dificultades que entraña elaborar una correcta descripción de dicho término, este artículo examina críticamente aquella concepción que imputa a la economía colaborativa un cierto potencial o pretensión emancipadora. Se mostrará que, paradójicamente, este discurso asume buena parte del utillaje conceptual de la teoría económica neoclásica estándar y por ello tiende a reproducir algunos de sus mismos errores. El artículo concluye sugiriendo que, para definir adecuadamente el concepto de la economía colaborativa, se requiere el empleo de la economía política como marco de análisis, más aun si lo que se pretende es una definición fértil, no sólo para abonar el debate conceptual, sino también los proyectos reales de emancipación política y económica.

Palabras clave: Economía y consumo colaborativo, economía neoclásica, emancipación, comunidad, mercados, limitaciones.

\section{ABSTRACT}

How the collaborative economy must be understood? Which methodologically operative as well as substantially informative definition should be employed? By identifying the difficulties in finding out a correct definition of such term, this paper criticaIly analyses the conception that infers to the collaborative economy a certain emancipatory aspiration or capacity. The paper shows that, paradoxically, such a discourse assumes much of the conceptual tools belonging to the stabdard neoclassical economic theory and, therefore, tends to reproduce some of mistakes. The conclusion suggests that, in order to properly define the concept of the collaborative economy, it is required the employment of the political economy as framework of analysis, even more if what it is intended is a fertile definition to foster, not only the discussion in the conceptual realm, but also the actual political and economic emancipatory initiatives.

Keywords: Collaborative economy and consume, neoclassical economy, emancipation, community, markets, limitations.

\footnotetext{
*Autor para correspondencia / Corresponding author: Bru Laín. Departamento de Sociología, Facultad de Economia y Empresa, Universidad de Barcelona, Av. Diagonal, 690-696, 08034, Barcelona.
}

Sugerencia de cita / Suggested citation: Laín, B. (2017). Algunas limitaciones en la comprensión de la economía colaborativa en sentido emancipador. Revista Española de Sociología, 26 (1), 109-114. 


\section{INTRODUCCIÓN}

Para abordar la llamada "economía colaborativa" (EC, en adelante) lo primero a resolver es su propio significado. La literatura suele referirse a ella como una suerte de nuevo modelo económico fundado sobre los principios de la interacción productor-consumidor, la conexión horizontal y la colaboración entre pares. A la vez, suele clasificarse de acuerdo a cuatro grandes subsectores. El más conocido es el consumo colaborativo (Airbnb, Uber o BlaBlaCar) que cuenta con una fuerte implantación, abarca dimensiones cuasi industriales y engloba tres grandes prácticas: la recirculación de bienes (Ebay o Craig's List) donde internet reduce los altos costes de transacción de los mercados secundarios; la optimización (compartir el coche en Zipcar 0 el sofá en Couchsurfing) posibilitando el acceso a recursos asequibles y generando pequeñas rentas adicionales; y el intercambio recíproco, paritario y multilateral (bancos del tiempo). El segundo subsector es la llamada producción colaborativa usualmente asociada a la lógica del software libre y del peer-to-peer (P2P) y que, según sus partidarios (Berardi, 2003; Hardt, 2010 y Boutang, 2012), supondría una suerte de revolución industrial democratizadora. El tercer grupo es el representado por el conocimiento abierto (open knowledge) sin el cual la propia EC perdería razón de ser. Su desarrollo, junto al uso intensificado de las TIC, permitiría tanto al saber abstracto como al aplicado replicarse y expandirse más allá del ámbito educativo convencional. El cuarto sector lo constituyen el sector de las finanzas colaborativas que pueden incluir la participación en capital empresarial (Kickstarter o Propser) del que se extraerían retornos en forma de beneficios, rentas o mediante la participación del accionariado de la empresa en cuestión; los sistemas de financiación individual P2P (LendingClub o Zopa); así como las plataformas de financiación crowdfunding (Lanzanos, Goteo o Verkamı) que, por lo general, se asocian más a iniciativas de carácter social y cultural ${ }^{1}$.

De este modo, el alto grado de penetración que la EC está teniendo en muchos ámbitos de la eco-

1 Para adentrarse en el debate sobre las plataformas crowdfunding como recursos comunes, véase: Rendueles y Sábada (2015). nomía — digamos— convencional, está suscitando una gran atención. Sin ir más lejos, la revista Forbes estimaba que en 2013 los ingresos globales distribuidos por la EC rondaban los 2500 millones de euros, con un $25 \%$ de crecimiento respecto al año anterior. Es una cifra importante, sin duda, aunque apenas represente el 0,2\% del PIB español. Igualmente relevante es que unos 800 millones de personas en el planeta se encuentren directa 0 indirectamente relacionadas con el sector de las cooperativas, aunque aparentemente esta cifra no genere el mismo interés. De todos modos, no pretendo aquí discutir la relevancia económica de la EC, sino más bien su conceptualización, en particular, su conceptualización como una suerte de economía que tiene la virtud superar las limitaciones y contradicciones que inherentemente conlleva el sistema de producción capitalista.

\section{¿CÓMO DEFINIR LA ECONOMÍA COLABORATIVA?}

Es aquí donde encontramos la primera dificultad. El problema con las definiciones y clasificaciones como la empleada anteriormente es que son poco operacionales para construir el "objeto formal" de estudio, puesto que no observan los criterios lógico-formales necesarios para realizar una correcta clasificación, Estos son, la no nulidad de ningún caso, la partición exhaustiva de todos los subgrupos y la exclusión entre todos los subconjuntos. En otras palabras, ¿debería o no incluirse la economía cooperativa como un subconjunto de la EC? ¿Hasta qué punto Airbnb, empresa privada convencional², y BlaBlaCar, red social para compartir el vehículo, caerían dentro del mismo subconjunto de la EC? ¿Pueden equipararse las prácticas de trabajar como conductor de Uber, la de prestar gratuitamente el sofá a través de Couchsurfing, 0

2 Airbnb está tasada en 7200 millones y 230 millones de beneficios en 2013. Sólo en Barcelona gestiona más de 12000 anuncios - frente las 9000 plazas turísticas con licencia-, un $60 \%$ de los cuales ofertan más de un piso a la vez, de lo que se deduce que lo hacen como negocio y no como modo de obtener ingresos extra. Agradezco a Lluís Torrens y Carlos Carrasco de IESE Business School los datos facilitados. 
compartir conexión mediante Wifi.net? Dados tales interrogantes, se me antoja que las definiciones y clasificaciones que usualmente manejamos de la EC suelen ser poco robustas metodológicamente y, por ello, están condenadas a ser poco o nada útiles como categorías de análisis empírico y conceptual.

Como lo primero para abordar un debate es definir adecuadamente el término analizado, será necesario identificar algunas de las problemáticas que entrañan este tipo de definiciones. La aportación de Piñeiro, Suriñach y Casadevante que acompaña este debate representa una relevante aportación en esta tarea. Por mi parte, propongo un ejercicio de abstracción. De un modo muy esquemático caracterizaré dos posturas más 0 menos representativas del debate sobre la EC. Por un lado, quienes la identificarían con un modo de producción, distribución y consumo más horizontal y participativo que, como apunta Albert Cañigueral (2014: 22), simplemente "quiere ofrecer más alternativas al sistema, más que ser una alternativa al sistema". La segunda postura, tal como sugiere Enrique Alonso en estas páginas, me parece más sugerente, puesto que vería en la $\mathrm{EC}$ un conjunto de prácticas económicas dotadas de una cierta capacidad o pretensión emancipadora 0 incluso anti 0 post-capitalista. Sin embargo, no busco discutir cuál de las dos concepciones es prescriptivamente más deseable, ni tan siquiera cuál la que mejor refleja la realidad. Lo que pretendo es más bien llamar la atención sobre la paradójica relación que se da entre ambas, más aun cuando aparentemente se erigen como contrarias u opuestas entre sí. El hecho de descubrir tal paradoja me permite esbozar algunas limitaciones que pueden aparecer en la defensa de una EC en clave emancipadora cuando de ella se emplea una definición poco robusta.

\section{TRES TESIS SOBRE LA ECONOMÍA COLABORATIVA EMANCIPADORA Y SUS LIMITACIONES}

\section{a. Modelos relacionales e instituciones sociales}

De acuerdo con la literatura antropológica (Fiske, 1992), las cuatro pautas relacionales observables en toda sociedad humana parecen agruparse bajo las relaciones de equivalencia, las de autoridad, las de igualdad y las de proporcionalidad. Es- tos patrones conductuales operarían como condiciones necesarias en la configuración de cualquier institución social. De este modo, para conformar una comunidad deberían observarse, por lo menos, relaciones de equivalencia. La institución estatal debería operar, como mínimo, bajo relaciones de autoridad. En el ámbito familiar, las relaciones de igualdad jugarían un papel fundacional, mientras que los mercados requerirían necesariamente de relaciones de proporcionalidad ${ }^{3}$. Es aquí donde se halla la primera confusión de la defensa de una EC de tipo emancipador.

Es habitual denominar "comunidad" al conjunto de individuos vinculados a cierta actividad asociada a la EC, como por ejemplo, la "comunidad Airbnb". Es igualmente frecuente (y confuso) el empleo de la metáfora de la "economía familiar" para referirse a la política macroeconómica del gobierno. Y es precisamente en el empleo de esta extendida metáfora — comunidad colaborativadonde aparece una doble confusión. El empeño en resaltar la centralidad de la "comunidad" en la EC, ¿expresa una voluntad o creencia de ver en ella la institución social responsable de toda actividad económica? Luego, si creemos que sí, que la comunidad o incluso la familia debieran relegar el rol del Estado y de los mercados como agentes económicos, entonces parecería lógico inducir que las pautas relacionales más asociadas a estas instituciones - la equivalencia y la igualdad — debieran regir toda actividad económica en detrimento de las relaciones de autoridad y proporcionalidad más propias del Estado y de los mercados.

3 Cabe advertir, que no se puede vincular una pauta conductual directamente con una institución social determinada. Los cuatro modelos relacionales son condición necesaria, pero no suficiente, para la emergencia de las distintas instituciones. De este modo, para hablar de familia deben observarse, por 10 menos, relaciones de igualdad; para el Estado, como mínimo, las de la autoridad; para los mercados, por lo pronto, las de proporcionalidad (a cada uno según su mérito) y, para la comunidad, las de equivalencia (a cada uno según sus necesidades, de cada cual según sus capacidades). Huelga decir que todos los patrones conductuales se hallan entremezclados en toda institución social. En ningún caso podemos afirmar que, por ejemplo en la familia, operen únicamente relaciones de igualdad, ni tampoco que el Estado solamente se configure de acuerdo al sometimiento autoritario del ciudadano, etc. 
El hecho de que las interacciones basadas en patrones de equivalencia y de igualdad puedan sernos moralmente más deseables, no implica que las relaciones de autoridad o de proporcionalidad no existan, y ni siquiera que no sean necesarias en el sí de todo sistema económico ${ }^{4}$. ¿Es factible, por ejemplo, un sistema de Seguridad Social gestionado íntegramente por comunidades? ¿Son plausibles una fiscalidad 0 una redistribución justas y equitativas sin el empeño de una autoridad estatal que legítimamente restrinja la evasión y el acaparamiento de determinados agentes sociales? ¿Y qué hay de la proporcionalidad?

Proponer una economía regida exclusivamente por un único modelo relacional —la equivalencia o la igualdad estrictas - refleja una concepción económica que confunde medios con fines. Creo que es muy difícil negar la utilidad de la autoridad en aquellas funciones que transciendan el ámbito de la comunidad, como el caso de la fiscalidad. Del mismo modo, puede reconocerse como justa aquella relación de proporcionalidad que sancione positivamente determinadas actividades a las que colectivamente imputemos un alto valor social, como por ejemplo, la experiencia 0 el sobre esfuerzo ${ }^{5}$.

La estructura de todo sistema económico se encuentra troquelada por una superposición de relaciones de autoridad y proporcionalidad, de dominación, de dependencias y de interferencias entre distintos sujetos y grupos sociales. La visión emancipadora de la EC tendería a desatender este hecho en favor de la ingenua creencia en su substitución mediante la emergencia espontánea de una suerte de red comunitaria colaborativa que permitiría compatibilizar grandes dosis de altruismo con un justo y eficiente modelo de justicia distributiva

4 Para debatir este punto, véase: Rendueles (2013: 98-102).

5 El debate sobre el "valor social" exige mucha más atención de la que puedo ofrecer aquí. Pero repárese en que abogar por que se "recompense proporcionalmente" el sobre esfuerzo bien puede referirse al reconocimiento de la actividad que realiza una gran parte de la fuerza de trabajo con "doble jornada laboral", esto es, el colectivo de mujeres que desempeña trabajo doméstico y asalariado simultáneamente. ¿Por qué no recompensar proporcionalmente este injusto sobre esfuerzo al que se hallan sometidas mediante un aumento salarial, por ejemplo? que potenciaría simultáneamente las relaciones de equivalencia y de igualdad.

\section{b. Estructura social y cognición humana}

Junto a esta creencia en la preeminencia de la comunidad como institución económica básica y del igualitarismo como patrón conductual preeminente en toda relación económica, aparece un segundo problema en la concepción emancipadora de la EC. Éste viene motivado por la pretensión de imputarle un carácter más o menos post-capitalista cuando, de hecho, esta concepción comparte parte del utillaje conceptual de la economía neoclásica estándar. Para ilustrar esta curiosa relación echemos mano de otro tópico. Un economista neoclásico argumentaría del siguiente modo: "dado que los agentes son racionales, si les dejamos en libertad para competir entre ellos, y dicha competición es perfecta, éstos lograrán un mercado eficiente y paretianamente optimizado en todas sus dimensiones". Seguiría el mismo proceder un hipotético defensor de la EC emancipadora, ceteris paribus: "dado que los agentes son colaborativos, si les dejamos en libertad para colaborar, y dicha colaboración es perfecta, entonces lograrán una economía colaborativa justa en todas sus dimensiones".

Repárese en que ambas locuciones son formalmente equivalentes. El problema radica en que no es que los individuos - racionales o colaborativos, esto ahora es irrelevante- dejados en libertad para competir (o colaborar), puedan lograr una economía competitiva (o colaborativa) óptima (o justa). La cosa funciona más bien al revés. La racionalidad instrumental y maximizadora del homo economicus solo sería plausible si se encontrara inserta de antemano en un mercado óptimamente competitivo donde el coste de la información fuera cero. El ideal emancipador de la EC incurriría en el mismo error: la existencia del sujeto perfectamente colaborativo sólo sería plausible si éste se encontrara de entrada en un entorno verdaderamente colaborativo donde el coste de dicha colaboración fuera cero o cercano a cero. En definitiva, la idea (o el ideal) del agente racional-maximizador, así como del colaborativo que actúa en base a información o colaboración a coste cero, presupone necesariamente una estructura social determinada de antemano. 
Regresamos así al punto anterior, ¿son realmente concebibles las motivaciones cognitivas y los distintos modelos relacionales de los agentes sin atender al entramado institucional en el cual se insertan? ¿Podemos creer en la cognición humana "colaborativa" como factor determinante en la constitución y desarrollo de los mercados sin reparar en las múltiples relaciones de dominación y los vínculos de dependencia que ya operan ex-ante en dichos mercados? Si no aceptamos el ideal del homo economicus como variable explicativa de los mercados capitalistas, tampoco deberíamos hacerlo en el caso de los agentes y los mercados colaborativos.

\section{c. Desinstitucionalización de la vida económica.}

Aparece aquí la tercera limitación de una EC emancipadora y que igualmente viene motivada por el empleo del utillaje neoclásico estándar. Si el grueso de la teoría económica y política clásica (desde Aristóteles hasta Marx) estuvo siempre interesada por la distribución del ingreso, del capital y de los recursos productivos - esto es, de la justicia distributiva - , fue porque la entendían como una suerte de precondición para la ulterior distribución de rentas y la formación de precios relativos. A los neoclásicos y neoliberales, por el contrario, les interesa la distribución de la renta, no como determinante sino como efecto derivado del proceso general de formación de rentas y precios dentro de los mercados. Para la economía clásica, la distribución de riquezas y de ingresos (y del poder de negociación que éstas confieren) queda determinada política y exógenamente desde fuera de los mercados (Bertomeu y Domènech, 2005). Para los neoclásicos, y me temo que para buena parte de la concepción emancipadora de la EC, la distribución de la riqueza y del ingreso se formaría y quedaría determinada endógenamente dentro de los mercados, en este caso, de tipo colaborativo.

La distribución de la riqueza es uno de los estadísticos que, como el de Gini, sirve para medir el grado de desigualdad distributiva de una sociedad determinada. Sin embargo, la distribución funcional de la renta aporta información adicional acerca de qué tipo es y cómo se distribuye dicha riqueza. El año 2013 fue la primera vez que en la economía española los beneficios empresariales y las rentas de capital superaron las salariales ${ }^{6}$. ¿Responde esto exclusivamente al proceso de formación de precios (de salarios, de bienes y de mercancías) dentro de los mercados, o por el contrario afectan otros factores exógenos y de carácter político, como la pérdida de la influencia sindical y la extinción de la negociación colectiva, la política fiscal o las sucesivas reformas laborales? Un neoclásico optaría por la primera respuesta. Un clásico —o por lo menos, quien no quisiera ser neoclásico al defender la EC en clave emancipadora- debería fijarse en la segunda explicación.

En efecto, al proclamar el carácter emancipador de la EC desde la óptica neoclásica estándar, existe el peligro de incurrir en dos errores. Primero, el más extremo, inferir que la simple presencia de las comunidades colaborativas es premisa suficiente para lograr una distribución más justa y equitativa de la riqueza. El segundo, más laxo, creer que la expansión de la EC es un factor necesario para avanzar hacia dicha justicia distributiva. Una y otra son hipótesis distintas, aunque forman parte de la misma perspectiva, a saber, aquella que prima una concepción meramente psicológica (y psicologizante) de los mercados, obviando la responsabilidad que tiene el entramado político-institucional (la presión sindical, la distribución de la propiedad, la tasa de acumulación y de especulación, la política fiscal, el poder de las distintas clases sociales, el régimen político, etc.) como factor causal determinante en la constitución de un diseño más o menos democrático y más o menos equitativo de justicia distributiva.

\section{CONCLUSIONES}

Como apunté al inicio, abordar el debate sobre la EC es una tarea harto compleja desde el punto de vista metodológico. A resultas de ello, una definición escasamente rigurosa es poco o nada útil para operacionalizar el análisis. El problema es que se vuelve sustancialmente imposible aunar bajo el

6 Según el INE de 2013, rentas de capital y beneficios sumaban el $46 \%$ del PIB, mientras que la participación salarial apenas rozaba el $44 \%$. 
mismo denominador prácticas colaborativas que nada tienen que ver entre sí. ¿Cómo y bajo qué parámetros se decide qué es EC? ¿Deberían incluirse por igual iniciativas tan dispares como Ebay, el couchsurfing o la cooperativa Somenergía? Y si así fuera, ¿caerían dentro del mismo subconjunto del consumo colaborativo o deberían clasificarse en distintos subsectores de la EC? Llamé también la atención sobre las limitaciones que entraña una hipotética defensa de la EC en clave emancipadora. Que esta concepción comparta parte de los argumentos de la teoría económica estándar la condena a incurrir en los mismos errores.

La colaboración económica es una práctica que se desarrolla a través de múltiples instituciones sociales de las que, a priori, no podemos excluir ni el Estado ni el mercado. Al decir de Marx y Polanyi, existen tres elementos constitutivos de cualquier sistema económico, a saber: la tierra, el dinero y la fuerza de trabajo. El modo en cómo políticamente se regulen, administren e incrusten estas tres "mercancías ficticias" en el entramado social, contribuirá a determinar el mayor o menor carácter "emancipador" de una economía. Con la EC pasa algo parecido. No es que ésta comporte necesariamente un avance hacia una mayor equidad social, sino que esta equidad vendrá determinada por el grado de mercantilización a la que estén sometidos los bienes empleados por la misma $\mathrm{EC}$, incluyendo los recursos productivos, el medio de intercambio y la fuerza de trabajo empleada.

A la luz de estas limitaciones, creo más adecuado comprender la EC como una suerte de prácticas económicas presentes en toda sociedad humana que, en función del contexto socio-político, pueden toman una forma institucional u otra y, por ello, un carácter más 0 menos democrático. Por ello, para imputar un carácter emancipador a la EC, es necesario ir más allá de las meras predisposiciones psicológicas de los agentes y tener en cuenta el diseño y la orientación política de las instituciones en las cuales se inserten y desarrollen dichas prácticas colaborativas. En otras palabras, si el fin es lograr una sociedad más justa y equitativa, no podemos limitar los medios a exclusivamente las comunidades colaborativas y las relaciones de igualdad y equivalencia. Requerimos pues, de la perspectiva de la economía política, no sólo para comprender cabalmente el fenómeno de la EC, sino para que ésta pueda desplegar su potencial en favor de mayores cuotas de emancipación, de justicia distributiva y, en definitiva, de democracia económica.

\section{AGRADECIMIENTOS Y FINANCIACIÓN}

Bajo la financiación del Consejo de Investiga-

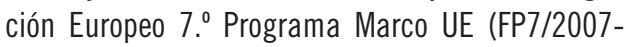
2013). Convenio ERC 249438-TRAMOD. Agradezco a Luís Enrique Alonso su invitación a participar de este monográfico, así como los valiosos comentarios que realizaron Marc Pradel, Edgar Manjarín, Ivan Miró, David Casassas y César Rendueles.

\section{REFERENCIAS BIBLIOGRÁFICAS}

Berardi, F. (2003). La fábrica de la infelicidad. Madrid. Traficantes de Sueños.

Bertomeu, M. J. y Domènech, A. (2005). El republicanismo y la crisis del rawlsismo metodológico (Nota sobre método y sustancia normativa en el debate republicano). Isegoría. Revista de Filosofía Moral y Política, 33, 51-75.

Boutang, Y. (2012). La abeja y el economista. Madrid. Traficantes de Sueños.

Cañigueral, A. (2014). Los retos de la economía colaborativa, en Economistas sin fronteras (eds.). Economía en colaboración, 12, 22-23.

Fiske, A. (1992). The Four Elementary Forms of Sociality: Framework for a Unified Theory of Social Relations. Psychological Review, 99 (4), 689-723.

Hardt, M. (2010). The Common in Communism. Rethinking Marxism: A Journal of Economics, Culture \& Society, 22 (3), 346-356.

Rendueles, C. (2013). Sociofobia. El cambio político en la era de la utopía digital. Madrid: Capitán Swing.

Rendueles, C. y Sábada, I. (2015). Los bienes comunes en un entorno de fragilidad social: el caso del crowdfunding, en Economistas sin fronteras (eds.). El procomún y los bienes comunes, 16, 42-47. 